\title{
A comprehensive survey of Retzius periodicities in fossil hominins and other great apes
}

Russell Hogg a, *, Rodrigo Lacruz ${ }^{\mathrm{b}}$, Timothy G. Bromage ${ }^{\mathrm{b}, \mathrm{c}}$, M. Christopher Dean ${ }^{\mathrm{d}, \mathrm{e}}$, Fernando Ramirez-Rozzi ${ }^{\text {}, ~ S e n t h i l ~ B a l a j i ~ G i r i m u r u g a n ~}{ }^{\mathrm{g}}$, Amanda McGrosky ${ }^{\mathrm{h}}$, Gary T. Schwartz ${ }^{\mathrm{h}}$

${ }^{a}$ Department of Rehabilitation Sciences, Florida Gulf Coast University, 10501 FGCU Blvd South, Ft. Myers, FL, 33965, USA

b Department of Basic Science and Craniofacial Biology, New York University College of Dentistry, 345 E. 24 ${ }^{\text {th }}$ St., New York, NY, 10010, USA

${ }^{\mathrm{c}}$ Department of Biomaterials and Biomimetics, New York University College of Dentistry, 345 E. $24^{\text {th }}$ St., New York, NY, 10010, USA

${ }^{\mathrm{d}}$ Centre for Human Evolution Research (CHER), Department of Earth Sciences, Natural History Museum, London, SW7 5BD, UK

e Department of Cell and Developmental Biology, University College London, Gower Street, London, WC1E 6BT, UK

${ }^{\mathrm{f}}$ Écoanthropologie, Musée de l'Homme, UMR 7206, 17, place du Trocadéro, Paris, 75116, France

${ }^{\mathrm{g}}$ Department of Mathematics, Florida Gulf Coast University, 10501 FGCU Blvd South, Ft. Myers, FL, 33965, USA

h Institute of Human Origins, School of Human Evolution and Social Change, Arizona State University, Tempe, AZ 85287, USA

* Corresponding author.

E-mail address: rhogg@fgcu.edu (R. Hogg)

\begin{abstract}
Recent studies have provided great insight into hominin life history evolution by utilitizing incremental lines found in dental tissues to reconstruct and compare the growth records of extant and extinct humans versus other ape taxa. Among the hominins, studies that have examined Retzius periodicity (RP) variation have come to contradictory conclusions in some instances. In order to clarify RP variation among hominins and better place this variation in its broader evolutionary context, we conduct the most comprehensive analysis of published RP values for hominins and great apes to date. We gathered all available data from the literature on RP data from extant humans, great apes, and fossil hominins, and assessed their variation using parametric and nonparametric analyses of variance. We also performed phylogenetic generalized least-squares (PGLS) regressions of RP data for these taxa as well as a larger set of hominoids for which RP data have been published against data for body mass, encephalization, and mean semicircular canal radius (SCR, a proxy for metabolic rate). Our results show that modern humans have a mean RP significantly differing from that of other hominins. Pongo also is significantly different from nearly all other taxa in all analyses. Our results also demonstrate that RP variation among hominins scales with respect to body mass, encephalization, and SCR similarly to other hominids, but that modern humans and Pongo stand out in this regard. Operating within the hypothesis that RP reflects autonomic biorhythms that regulate multiple life history variables, our results reinforce the idea that Homo sapiens has evolved a life history distinct from other hominins, even from other members of Homo, and suggest that many of these life history differences may be driven by hypothalamic output from the brain.
\end{abstract}


Keywords: Life history; Enamel microstructure; Striae of Retzius; Incremental lines; HaversHalberg oscillation

\section{Introduction}

Anthropologists have long observed that anatomically modern humans have an unusual life history compared to their closest living relatives, the great apes (e.g., Schultz, 1960). Modern humans have a relatively higher energy budget and reproductive output ('fast' life history attributes), coupled with a paradoxically long juvenile period and lifespan ('slow' life history attributes; Harvey and Clutton-Brock, 1985; Leigh, 2004, 2012; Reiches, et al. 2009; Isler and van Schaik, 2012; Pontzer, 2012; Schwartz, 2012). These features are key components of a derived life history profile that makes Homo sapiens unique. Therefore, much attention has been devoted to illuminating the selective forces that led to this unusual life history (Smith and Tompkins, 1995; Kaplan et al., 2000; Leigh, 2001, 2004, 2012; Pontzer, 2012).

Increasingly, this avenue of research takes advantage of histological techniques to study in detail the growth processes of teeth and bones, and to relate these direct growth data to models of human life history evolution (e.g., Bromage and Dean, 1985; Dean and Beynon, 1991; Dean, 1995, 1998, 2000; Schwartz et al., 2001, 2005; Lacruz et al., 2008; Hogg et al., 2015, 2017; Smith et al., 2015, 2018). These studies take advantage of the fact that dental tissues preserve permanent growth lines as part of their structure (Fig. 1). These growth lines can be used to reconstruct a chronology of growth in a manner similar to dendrochronology (e.g., Bromage and Dean, 1985; Dean and Beynon, 1991; Dean 1995, 1998, 2006; Schwartz et al., 2001; Lacruz et al., 2008; Schwartz, 2012; Smith et al., 2015). Some studies have demonstrated that aspects of modern human dental growth, such as crown extension rates, crown formation times, and M1 ages at emergence may differ from those of other living great apes, and from australopiths (e.g., Bromage and Dean, 1985; Lacruz and Bromage, 2006; Kelley and Schwartz, 2012; Schwartz, 2012), although there may be substantial intraspecific variation in these variables (Smith et al., 2015). Within Homo in particular, there are conflicting reports; for example, different studies disagree as to whether dental development in Neanderthals differs substantively from that of modern humans (e.g., Smith et al., 2010; Rosas et al., 2017).

One specific area where studies disagree is in regard to differences in variation of Retzius periodicity (RP, sometimes referred to as 'repeat interval' or simply as 'periodicity;' see Hogg et al., 2015) among modern humans, hominins, and hominids. RP refers to the number of days between the deposition of successive long-period growth lines in teeth. Within tooth enamel, these long-period lines are referred to as striae of Retzius, whereas they are termed Andresen lines in dentine. RP varies among individuals within a species, and among species, and is quantified by counting the number of daily growth lines (known as cross-striations in enamel; von Ebner lines in dentine) between successive long-period lines (Fig. 1).

$\mathrm{RP}$ is an important variable for understanding life history evolution, as several studies have suggested that it is a histological manifestation of a neuroendocrine biorhythm, the HaversHalberg oscillation (HHO; Bromage et al., 2009, 2012; Hogg et al. 2017). The HHO hypothesis is based on early observations by Dean (1995) and Dean and Scandrett (1996) that there might be a correlation between RP and body mass, and that RP may also be tied to autonomic biorhythms as reflected in heart rate oscillation (Appenzeller et al., 2005). The HHO is hypothesized to play a role in regulating the overall pace of mammalian life history, and as a general rule correlates strongly with body mass and metabolic rate among anthropoid primates (Bromage et al., 2009; Bromage et al., 2012; Hogg et al., 2015; Hogg et al., 2017). The HHO potentially regulates 
metabolic output, growth rates, and lifespan among other life history characteristics (Bromage and Janal, 2014; Bromage et al., 2009, 2012, 2015, 2016; Hogg et al., 2015, 2017).

The HHO hypothesis argues that cell proliferation and activity, cellular metabolism, and cell growth, are influenced by oscillations in sympathetic output originating in the hypothalamus of the brain (Bromage et al., 2009; Hogg et al., 2017). There is ample evidence underlying the physiology of the HHO in the literature on bone-energy homeostasis, recently reviewed by Hogg et al. (2017). Briefly, the leptin-sympathetic-osteocalcin feedback loop (Hogg et al., 2017) participates with hypothalamically controlled biorhythms that act as metronomes pacing cellular activity. The central biorhythm, the HHO, is correlated with cell proliferation rates, as was shown by demonstrating a correlation between osteocyte density and body mass interspecifically (Bromage et al., 2009; Hogg et al., 2017). HHO also implicates metabolic rate as evidenced by correlations between RP and metabolic rates that are tissue-specific (Bromage and Janal, 2014, Hogg et al., 2017). Finally, direct assessments of metabolite levels revealed clear oscillations over a period of several days in pigs (Bromage et al., 2009, 2015, 2016; Bromage and Janal, 2014). Moreover, by affecting cellular activity in osteoblasts, ameloblasts, and odontoblasts that lay down bone and dental tissues, the hypothesis argues that the HHO rhythm is permanently encoded and therefore readable as growth increments (e.g., striae of Retzius) in mineralized tissues. Importantly, if the HHO is part of a neuroendocrine feedback loop affecting cell proliferation, metabolism, and growth, then variations in HHO periodicity could have been evolutionary targets because selective pressures on the HHO modulated life history. Therefore, RP data have great potential to provide important insights into life history evolution. While there is much work to be done to further verify the HHO hypothesis - for example, direct experimental evidence that HHO-related biorhythms such as RP are a result of autonomic oscillation has not yet been sought - and identify the molecular networks involved, the HHO remains a powerful model to advance our understanding of life history evolution (e.g., Bromage et al., 2009; Hogg et al., 2015) despite it also being true that life history features have the ability to vary independently (e.g., Hogg et al., 2015, 2018). Again, readers are referred to Hogg et al. (2017) for a more complete review on the physiological literature underlying the HHO hypothesis.

Several studies have sampled RP among modern humans, fossil hominins, and great apes. Bromage et al. (2009) collated hominin RP data from the literature, estimating a range between 6 and 9 days $(n=33$ specimens across Australopithecus anamensis, Australopithecus afarensis, Australopithecus africanus, Paranthropus aethiopicus, Paranthropus boisei, Homo rudolfensis, Homo erectus, and Homo ergaster) and found a strong correlation between RP and reconstructed body mass $(\mathrm{r}=0.87, p<0.01)$. This study noted that RP variability in modern humans is unusually high, in contrast to findings for other hominins (Lacruz et al., 2008). Smith et al. (2015) sampled 25 fossil hominins, including A. anamensis, A. africanus, Pa. robustus, and South African "Homo," and compared RP values in their sample with RP data from the literature for $H$. erectus, $H$. neanderthalensis, fossil and extant $H$. sapiens, Pan troglodytes, and Gorilla gorilla. In contrast to the conclusions of Lacruz et al. (2008) and Bromage et al. (2009), they found that fossil hominins do not show a smaller range in RP or a lower average RP when compared to modern humans. Using Mann-Whitney tests, they reported that there was no significant difference in RP between modern humans and australopiths. These studies utilized different data sets with different samples, and employed different analytical protocols, and as a result, it is perhaps not surprising that they reached different conclusions.

Fortunately, there has been a steady accumulation of published hominin and hominoid RP data over the last twenty years (e.g., Bromage et al., 2007, 2009; Macchiarelli et al., 2006; 
Lacruz et al., 2008, 2012; Lacruz and Ramirez-Rozzi, 2010; Smith et al., 2007a, b, 2009, 2010, 2015; Ward et al., 2001). This enables a comprehensive look at RP variation in these groups, which as mentioned above, is important because of the potential insights into life history it provides via the lens of the HHO (Hogg et al., 2017). We therefore performed a comprehensive literature search to gather RP values published for extant and extinct individuals within Hominidae, inclusive of extant and extinct taxa. In so doing, we asked four primary questions:

(1) What are the overall patterns of mean RP across the hominid family? (2) Are there significant differences in mean RP among hominins and other hominids, namely Gorilla, Pan, and Pongo?

(3) Are there significant differences in mean RP between modern humans and other hominins?

(4) How is RP correlated with body mass, brain mass, and metabolic rates among hominins and hominids?

\section{Materials and methods}

We gathered RP data from the literature for 1492 individuals across 19 hominid species, 1194 of these from modern humans and 298 from the remaining species set (Table 1). Since a recent hypothesis posits that RP may change between deciduous and permanent teeth (Mahoney et al., 2016), we included only data from permanent teeth. Where possible, we computed summary statistics for males and females separately, and also included species mean values. See Table 1 for range, mean, mode, and sample size for each species, as well as data sources, and Table 2 for further descriptive statistics of select subgroups. The complete dataset is available as a spreadsheet in Supplementary Online Material (SOM) Table S1.

Due to small and unequal sample sizes, as well as the non-normal distribution of most of our dataset, our primary analysis consisted of nonparametric Kruskall-Wallis tests and Dunn's post hoc tests; non-parametric tests also minimize the impact of outliers as they do not take into account differences in magnitude among the sample set. As our between-group analyses of variance in RP were designed to seek out statistically significant subsets of hominid species and were not used to ascertain whether differences were confounded by phylogeny or other factors, we did not employ phylogenetic controls here. Tests were performed using IBM SPSS Statistics v. 25.0 (IBM Corp., 2017), and in R v.3.5.2 (R Core Team; 2018).

One potential confounding factor in our dataset is the overwhelming disproportion of our modern human sample size as compared to the sample sizes of all other hominids in our study. Moreover, within our modern human sample, there is a highly disproportionate contribution from different geographic areas, such that running statistical analyses on the complete, unmodified sample would likely yield results that do not accurately reflect the real biological variation of our populations. Accordingly, for all analyses involving modern humans, we resampled the dataset by coding every specimen according to geographic origin, and then randomly incorporating 40 individuals from each region into the analysis. If a particular region had a population of less than 40 in our dataset, we simply included the entire population from that region. We then bootstrapped this resampling through 5000 iterations, and performed Kruskal-Wallis and Dunn's analyses on the bootstrapped results. This process reduced the size difference between our modern human sample and all other samples, and also prevented any one geographic region in our human dataset from having an outsized effect.

Another potential confounding factor in dealing with RP data is measurement error in assessments of RP for individual specimens. It is recognized that determining RP for certain specimens can be particularly difficult, such that different observers will record different values when determining the number of days between secretion of successive striae of Retzius (Smith et 
al., 2007a). This is of particular concern for a study such as ours, which is compiling data from multiple studies in the literature. A reliable method for decreasing measurement error is the twopronged approach advocated by Schwartz et al. (2001). In this method, RP is assessed by two methods which are cross-checked against each other. In the first method, RP is assessed visually by counting cross-striations between successive striae of Retzius in an image of a tooth. In the second method, the mean of the measurements between successive striae of Retzius in a particular tooth region are divided by the mean daily secretion rate (DSR) for that same region. For an RP to be counted as accurate, the results of both methods must match for each individual. Schwartz et al. (2001) demonstrated a less than $3 \%$ interobserver error using this method. That said, fossils can provide extra difficulty with regard to presenting readily interpretable anatomy, different imaging methods were used by different sources in our study, and, more importantly, not all data sources for our study used the two-pronged method, instead opting only for direct counting or via estimation using DSR. Therefore, it is important to account for measurement error in our study in some way. We opted to assess the impact of error through a random error test, wherein measurement errors of $-2,-1,0,1$, and 2 days were sampled uniformly and added to the observations for within each genus and species depending on the test. An F-statistic and a Kruskal-Wallis statistic was obtained per run. The above procedure was repeated 10,000 times to obtain sampling distributions of the statistics.

To further immunize our analyses against error resulting from our comparison of several small sample sets drawn from fossil material against much larger samples drawn from extant taxa, we also ran a bootstrapping analysis of the ANOVA F statistics, resampling the dataset with replacement and repeating this process for a total of 1000 iterations. This permits the calculation of a $95 \%$ confidence interval for the $\mathrm{F}$ statistic from the sampling distribution.

It is also important to consider recent revisions of Pongo systematics and our inclusion of fossil Pongo teeth in our sample. It is difficult to impossible to determine whether RP values from all individuals previously reported as Po. pygmaeus may in fact belong to one of the newly erected Pongo species. Moreover, the fossil Pongo specimens we included have been attributed to Po. pygmaeus weidenreichi (Hu et al., 2012) but the taxonomy of these specimens is by no means certain. However, in both parametric and non-parametric pairwise comparisons, there is no significant difference among Pongo samples from different sources in our study, and no significant difference between fossil and extant Pongo $(p<0.0001$ in all tests). Therefore, we have opted to include all Pongo specimens in one sample in both genus and species level analyses.

For our correlation analyses, we wanted to expand upon results of prior studies (Smith, 2008; Bromage et al., 2009; Hogg et al., 2015) and examine how life-history variables correlate with RP among hominins in light of the HHO model. Additionally, we were interested in determining how much of the variation in RP across our sample is correlated with the degree of phylogenetic relatedness in our included taxa. Therefore, we gathered species mean data for body mass, index of cranial capacity (ICC), and relative semicircular canal radius (SCR, a proxy variable for metabolic rate), following the protocols of Hogg et al. (2015), and regressed species mean RP data against these metrics using standard phylogenetic generalized least-squares (PGLS) regression. Relative SCR is an index value for SCR that corrects for body mass following the regression statistic for primates given in Spoor et al. (2007): Relative SCR = mean $\mathrm{SCR}(\mathrm{mm}) /$ body $\operatorname{mass}(\mathrm{g})^{0.14}$; see Table 1 for data and sources. We incorporate SCR here as a proxy variable for metabolic rate based on a very strong and significant correlation between SCR and basal metabolic rate (BMR) in anthropoid primates identified by Hogg et al. (2015). This 
finding was in turn built on a correlation between SCR and activity levels in primates identified by the studies of Spoor et al. (2007) and Walker et al. (2008); founded upon this evidence, Hogg et al. (2015) found SCR to be an effective proxy variable for BMR in their study of RP variation in lemurs, and we follow their protocols here.

To evaluate the relationship between body mass and RP, having a wide range of body masses in the analysis is helpful for revealing any patterns that may be present within the sample. Having a wide range of body masses is also helpful to identifying where the sample lies within the broader context of variability of its parent taxon. Therefore in addition to our hominin and hominid sample, we included data for non-hominid hominoid species. These additional hominoid data were drawn from Hogg et al. (2015). Species' mean RP were regressed against body mass, ICC, and SCR data using the 'caper' package's (Orme et al., 2010) PGLS models in R. Trees were constructed using data from 10k trees for extant taxa (https://10ktrees.nunn-lab.org) and Dembo et al. (2016) for extinct taxa. Phylogenetic signal (Pagel's $\lambda$ ) was estimated using maximum likelihood. Though the sample contained at most 22 species, only PGLS regressions were run since PGLS results mirror ordinary least-squares (OLS) results if there is no phylogenetic signal in the residual structure of the data (Symonds and Blomberg, 2014).

\section{Results}

Figure 2 provides a boxplot illustrating characteristics of RP variation in the various species of our sample. With regard to differences between taxa Kruskal-Wallis tests show that when analyzing our sample both by genus and by species, significant differences are present $(p<$ 0.01 for both; Table 3 ). Bootstrapping analyses, to correct for sample size differences, are also available in Table 3. Our random error study demonstrated that the distribution had means well into the rejection region for the null hypothesis for either test and the entire distribution was over the rejection region indicating differences in RP for both genus as well as species. The distribution of $p$-values were less than 0.01 in every randomized repetition (Table 4). This suggests that interobserver/intraobserver error is not having a significant impact on our results.

The results of post hoc Tukey and Dunn's analyses, used to diagnose significant subsets, are detailed in Tables 5 and 6. At the species level (Table 5), the most obvious pattern is that our Pongo spp. dataset differs significantly from every other species except for $H$. rudolfensis and fossil $H$. sapiens; even for these species the $p$-value is close to significance. Gorilla gorilla stands out the next, differing significantly from five other species. Beyond this, no clear pattern is evident. In analyses at the genus level (Table 6), where sample sizes of non-modern humans are much increased, it immediately stands out that modern human RP differs significantly from all genera (including fossil Homo), with the lone exception of Gorilla. This is a marked contrast from the interpretations of Smith et al. (2015), who implied that RP variability among modern humans is not significantly different than that of other hominins, based on the similarity of the $\mathrm{RP}$ ranges demonstrated by modern humans and other hominins, and the lack of significant difference between modern humans, $A$. africanus, and $P a$. robustus in their sample. Other members of Homo do not seem to display significant differences compared to other hominins (Table 6), which agrees with results from previous studies (Lacruz et al., 2008; Smith et al., 2015). Also, Pongo once again differs significantly from all other genera, as does Gorilla (with the exception of modern humans).

PGLS regressions of species mean RP on body mass do not show a strong or significant relationship among hominins (slope $=0.06$, adj. $\mathrm{R}^{2}=0, p=0.43$; Table 7), nor do regressions of $\mathrm{RP}$ on SCR (slope $=1.04$, adj. $\mathrm{R}^{2}=0.26, p=0.11$; Table 7). However, there is a significant 
correlation between RP and ICC for this sample (slope $=0.17$, adj. $\mathrm{R}^{2}=0.42, p=0.03$; Table 9). The phylogenetic signal of the three hominin models is low $(\lambda=0)$, which indicates that phylogeny has very little effect on the error structure of the hominin-only data. The low correlation between body mass and RP among hominins masks some interesting biological relationships between these two variables for some parts of our sample: within hominoids as a whole, there is a significant correlation between species mean RP and body mass (PGLS slope $=$ 0.12 , adj. $\mathrm{R}^{2}=0.38, p<0.01$; Table 7). Interestingly, $\mathrm{SCR}$ also has a significant correlation with $\mathrm{RP}$ in this group (slope $=-1.79$, adj. $\mathrm{R}^{2}=0.45, p<0.01$; Table 7 ). For both body mass and SCR, the hominins that are included in the models cluster closely to the regression line for the entire hominid group and fall within the $95 \%$ confidence interval. Hominins, then, feature as fairly typical members of their family with regard to the relationship of species mean RP with body mass and SCR, respectively. Though the phylogenetic signal of the three hominoid models is high (Table 7), $\lambda$ estimates are sensitive to sample size (Freckleton et al., 2002) and all $\lambda$ 95\% confidence intervals, with the exception of that of the RP ICC model, include 0 in the lower bound. Interestingly, hominoid RP does not significantly correlate with ICC when phylogenetic relationships are taken into account (slope $=0.05$, adj. $\mathrm{R}^{2}=0.01, p=0.73$; Table 7; Figs. 3 and 4). However, given that SCR has been shown to be a reliable predictor of metabolic rate, under the HHO model of RP biology the relationship between SCR and RP is not surprising. The relationship between body mass and RP is also expected, since body mass has long been demonstrated to be a key determinant of metabolic rate, for example as laid out in Kleiber's law (Kleiber, 1932), and since body mass, bone metabolism, and energy homeostasis are known to be closely connected physiologically, via neuroendocrine control mechanisms (Hogg et al., 2017).

\section{Discussion}

With regard to our first and second question about differences in RP variation across the hominids, the evidence suggests that, firstly, the hominins as a group (exclusive of modern humans) seem to share patterns of RP variation (this term used in the vernacular sense) that are different from those of the great apes, i.e., Pan, Gorilla, and Pongo, since all three hominin genera differ significantly in RP from each of the great ape genera, but do not differ significantly among themselves. If RP is indeed tied to life history evolution as the HHO hypothesis posits, this may reflect evidence of differing life history patterns in hominins as compared to the great apes. Secondly, we see these differences extended among the great apes themselves, in that Pan, Gorilla, and Pongo all significantly differ from each other with regard to RP.

With regard to our third question, regarding evolution of RP among modern humans, $H$. sapiens does not demonstrate many significant differences from other taxa in our species-level analyses. However, much of this is likely due to the effect of small sample sizes among our fossil taxa; when redoing species-level analysis for modern humans by removing species where $\mathrm{n}<11$, bootstrap values and confidence intervals changed markedly, demonstrating that the specieslevel analysis is subject to sample size effects despite our best efforts to control for them. In our analysis comparing modern humans to other taxa at the genus level, where sample sizes are larger and results therefore more reliable, we show that modern humans do indeed differ significantly from other hominins in general, showing significant differences compared to Australopithecus and Paranthropus, as well as from the remainder of the genus Homo. This is to be expected, since it is quite well documented that modern human life history differs from that of all australopiths and early Homo (for discussion, see Kelley and Schwartz, 2012). If RP is driven by and reflective of the $\mathrm{HHO}$ as a periodicity in hypothalamic output and evolution in energy 
homeostasis (Hogg et al., 2017), the significant differences in RP between modern humans and other hominins, as well as between fossil Homo with respect to other hominins, suggest that RP patterns reflect derived patterns of life history for $H$. sapiens. Interestingly modern human mean RP does not differ significantly from Gorilla, a similarity deserving of further study.

Interestingly, the Xujiayao hominin has a periodicity higher than the mean of all other hominins; when viewed within the context that other dental development features in this specimen, such as crown formation time, are well within the range of modern humans, this reinforces the interpretation that this population may have had a very human-like life history (Xing et al., 2019). Indeed, a computed Z-score for the RP of this specimen, when analyzed in the context of our modern human dataset, sits at 1.58, demonstrating a fairly high value even as compared with modern humans. Compared to all other hominins, the Z-score for the Xujiayao hominin is even higher at 2.18, reinforcing the idea that it is more like modern humans than other hominins with regard to RP (even though this applies only to this specimen itself, and we can make no arguments with regard to its population in general).

Pongo is another interesting case, standing out in all analyses from all other genera, and also differing from more taxa than any other in species-level analyses. Pontzer (2017) convincingly argued that Pongo exhibits an unusual metabolic strategy for hominoids, with reduced BMR and lower daily energy requirements that may represent adaptations to crashes in food availability. Given the assumption that RP is reflective of metabolic biology driven by the hypothalamus, via the $\mathrm{HHO}$, our analysis here may help provide direction for elucidating how and why Pongo has evolved its highly derived metabolism, just as our results may help provide insight as to how and why modern humans have evolved such a derived life history.

Lastly, with regard to our fourth question, combining all published records available to date for RP, our results support prior studies in showing that hominids and hominins seem to follow correlations between RP and body mass, ICC, and SCR that are exhibited in anthropoid primates more generally (Bromage et al., 2009; Hogg, 2010; Hogg et al., 2015); that is, while we do not find a correlation between RP and body mass within the total hominin sample, hominoids as a whole do exhibit a significant relationship in this regard. Hominins fall close to the regression line for hominoids overall, and fall within the $95 \%$ confidence interval for hominoids as whole. Therefore, hominins seem to behave as stereotypical hominoids as far as RP and body mass correlations are concerned. It is also of interest that among hominoids SCR, which is a reasonable proxy for (Hogg et al., 2015), seems to exhibit the strongest correlation with RP. Not surprisingly, given the significant differences in RP variation between Pongo and all other species in our ANOVA sample, this taxon falls farthest from the overall regression line. Moreover, although Pongo is an outlier in regressions of RP with body mass and ICC, it does not particularly stand out as an outlier in regressions for SCR. This suggests that Pongo RP, and by extension its HHO biology, are not unusual.

\section{Conclusions}

By compiling the most extensive sample of RP values for hominids to date, we are able to elucidate important patterns of variation that address outstanding issues in hominin life history based on RP values as key biological markers. In sum, interpreted via the lens of the HHO model, RP data suggest that the modern humans may exhibit a life history that differs from that of other hominins and hominids, tied to hypothalamic regulation. They also suggest that hominins in general may have evolved a specialized life history compared to other hominids, and that Gorilla, Pan, and Pongo may also all exhibit derived HHO biologies as reflected in RP. For 
example, the previously observed high metabolic rate and reproductive throughput of modern humans (Pontzer 2012, 2017) may be a consequence of our unique HHO biology, and reflected in our different RPs. Of course, more information on the physiology of the HHO will be needed to tease apart the impact of HHO evolution upon human life history evolution in greater detail, and of course RP and HHO can only serve as indicators that some aspects life history evolution are different among particular taxa, since life history biology is modular physiologically and not $100 \%$ driven by the HHO. In any case, the data suggest that evolution in body mass and metabolic rate may be major driving factors underlying RP and therefore $\mathrm{HHO}$ variation. For example, Pongo stands out as being highly derived in terms of RP variation, but this variation may simply be an expression of its unique underlying metabolic biology.

\section{Acknowledgements}

R.S.L. and T.G.B. would like to acknowledge The Leakey Foundation, P.A.S.T in South Africa, The National Museums of Kenya and Ethiopia, and the University of the Witwatersrand for funding and access to fossil material.

\section{References}

Appenzeller, O., Gunga, H.C., Qualls, C., Furlan, R., Porta, A., Lucas, S., Hecker, A., Kirsch, K., Costa-Junqueira, M., Guillen, S., Sander, M., Schneider, T., Blottner, B., 2005. A hypothesis: autonomic rhythms are reflected in growth lines of teeth in humans and extinct archosaurs. Autonomic Neuroscience 117, 115-119.

Arsuaga, J.L., Martínez, I., Gracia, A., Carretero, J.M., Carbonell, E., 1993. Three new human skulls from the Sima de los Huesos middle Pleistocene site in Sierra de Atapuerca, Spain. Nature 362, 534-537.

Beynon, A.D., Dean, M.C., Reid, D.J., 1991. Histological study on the chronology of the developing dentition in gorilla and orangutan. American Journal of Physical Anthropology 86, 189-203.

Bromage, T.G., Dean, M.C., 1985. Re-evaluation of the age at death of immature fossil hominids. Nature 317, 525-527.

Bromage, T.G., Lacruz, R., Perez-Ochoa, A., Boyde, A., 2007. Portable confocal scanning optical microscopy of Australopithecus africanus enamel microstructure. In: Bailey, S., Hublin, J.J. (Eds.), Dental Perspectives on Human Evolution: State of the Art Research in Dental Paleoanthropology. Springer, New York, pp. 193-209.

Bromage, T.G., LaCruz, R., Hogg., R., Goldman, H., McFarlin, S.C., Warshaw, J., Dirks, W., Perez Ochoa, A., Smolyar, I., Enlow, D.H., Boyde, A., 2009. Lamellar bone is an incremental tissue reconciling enamel rhythms, body size, and organismal life history. Calcified Tissue International 84, 388-404.

Bromage, T.G., Hogg, R.T., Lacruz, R.S., Hou, C., 2012. Primate enamel evinces long period biological timing and regulation of life history. Journal of Theoretical Biology 305, 131144.

Bromage, T.G., Idaghour, Y., Lacruz, R.S., Crenshaw, T.D., Ovsiy, O., Rotter, B., Hoffmeier, K., Schrenk, F., 2015. The swine plasma metabolome chronicles "many days" biological timing and functions linked to growth. PLoS One 11, e0145919.

Bromage, T.G., Juwayeyi, Y., Katris, J.A., Gomez, S., Ovsiy, O., Goldstein, J., Janal, M.N., Hu, B., Schrenk, F., 2016. The scaling of human osteocyte lacuna density with body size and metabolism. Comptes Rendus Palevol 15, 3-39. 
Collard, M., 2002. Grades and transitions in human evolution. Proceedings of the British Academy 106, 61-100.

Dean, M.C., 1995. The nature and periodicity of incremental lines in primate dentine and their relationship to periradicular bands in $\mathrm{OH} 16$ (Homo habilis). In: Moggi-Cecchi, J. (Ed.), Aspects of Dental Biology: Paleontology, Anthropology, and Evolution. Angelo Pontecorboli, Florence, pp. 239-265.

Dean, M.C., 1998. A comparative study of cross striation spacings in cuspal enamel and of four methods of estimating the time taken to grow molar cuspal enamel in Pan, Pongo, and Homo. Journal of Human Evolution 35, 449-462.

Dean, M.C., 2000. Progress in understanding hominoid dental development. Journal of Anatomy 197, 77-101.

Dean, M.C., 2006. Tooth microstructure tracks the pace of human life history evolution. Proceedings of the Royal Society B 273, 2799-2802.

Dean, M.C., Beynon, A.D., 1991. Histological reconstruction of crown formation times and initial root formation times in a modern human child. American Journal of Physical Anthropology 86, 215-228.

Dean, M.C., Beynon, A.D., Thackaray, J.F., Macho, G.A., 1993. Histological reconstruction of dental development and age at death of a juvenile Paranthropus robustus specimen, SK 63, from Swartkrans, South Africa. American Journal of Physical Anthropology 91, 401419.

Dean, M.C., Scandrett, A.E., 1996. The relation between long-period incremental markings in dentine and daily cross-striations in enamel in human teeth. Archives of Oral Biology 41, 233-241.

Dembo, M., Radovcic, D., Garvin, H.M., Laird, M.F., Schroeder, L., Scott, J.E., Brophy, J., Ackermann, R.R., Musiba, C.M., de Ruiter, D.J., Mooers, A., Collard, M., 2016. The evolutionary relationships and age of Homo naledi: An assessment using dated Bayesian phylogenetic methods. Journal of Human Evolution 97, 17-26.

FitzGerald, C.M., 1998. Do enamel microstructures have regular time dependency? Conclusions from the literature and a large-scale study. Journal of Human Evolution 35, 371-386.

Freckleton, R.P., Harvey, P.H., Pagel, M., 2002. Phylogenetic analysis and comparative data: A test and review of evidence. The American Naturalist 160, 712-726.

Harvey, P.H., Clutton-Brock, T.H., 1985. Life history variation in primates. Evolution 39, 559581.

Hogg, R.T., 2010. Dental microstructure and growth in the cebid primates. Ph.D. Dissertation, City University of New York.

Hogg., R.T., Bromage, T.G., Reid, D.J., 2014. Biorhythm variations underlying the evolution of human life history: Evidence from tooth and bone histology. American Journal of Physical Anthropology 150 S58, 143.

Hogg, R.T., Godfrey, L.R., Schwartz, G.T., Dirks, W., Bromage, T.G., 2015. Lemur biorhythms and life history evolution. PLoS One 10, e0134210.

Hogg, R.T., Bromage, T.G., Goldman, H.M., Katris, J.A., Clement, J.G., 2017. The HaversHalberg oscillation cycle and bone metabolism. In: Percival, C.J., Richtsmeier, J.T. (Eds.), Building Bones: Early Bone Development in Anthropology. Cambridge University Press, Cambridge, pp. 254-280.

Hogg, R.T., Hu, B., Bromage, T.G., 2018. Histology of dental long-perio biorhythms in Canis familiaris. Journal of Anatomy 233, 618-624. 
Hu, R., Zhao, L., Wu, X., 2012. Periodicity of Retzius lines in fossil Pongo from South China. Chinese Science Bulletin 57, 790-794.

IBM Corp, 2017. IBM SPSS Statistics for Windows. Version 25.0. IBM Corp., Armonk, NY.

Isler, K., Van Schaik, C.P., 2012. Allomaternal care, life history, and brain size evolution in mammals. Journal of Human Evolution 63, 52-63.

Kaplan, H., Hill, K., Lancaster, J., Hurtado, A.M., 2000. A theory of human life history evolution: diet, intelligence, and longevity. Evolutionary Anthropology 9, 156-185.

Karaaslan, H., Seckinger, J., Almabrok, A., Hu, B., Dong, H., Xia, D., Dekyi, T., Hogg, R.T., Zhou, J., Bromage, T.G., in press. Enamel multidien biological timing and body size variability among individuals of Chinese and Tibetan origins. Annals of Human Biology.

Kelley, J., Schwartz, G.T., 2012. Life-history inference in the early hominins Australopithecus and Paranthropus. International Journal of Primatology 33, 1332-1363.

Kleiber, M., 1932. Body size and metabolism. Hilgardia 6, 315-351.

Lacruz, R.S., Bromage, T.G., 2006a. Appositional enamel growth in molars of South African fossil hominids. Journal of Anatomy 209, 13-20.

Lacruz, R.S., Ramirez-Rozzi, F., Bromage, T.G., 2006b. Variation in enamel development of South African fossil hominids. Journal of Human Evolution 51, 580-590.

Lacruz, R.S., Dean., M.C., Ramirez-Rozzi, F., Bromage, T.G., 2008. Megadontia, striae periodicity and patterns of enamel secretion in Plio-Pleistocene fossil hominins. Journal of Anatomy 213, 148-158.

Lacruz, R.S., Ramirez Rozzi, F.V., 2010. Molar crown development in Australopithecus afarensis. Journal of Human Evolution 58, 201-206.

Lacruz, R.S., Ramirez Rozzi, F.V., Wood, B.A., Bromage, T.G., 2012. Brief communication: Molar development and crown areas in early Australopithecus. American Journal of Physical Anthropology 148, 632-640.

Leigh, S.R., 2001. Evolution of human growth. Evolutionary Anthropology 10, 223-236.

Leigh, S.R., 2004. Brain growth, life history, and cognition in primate and human evolution. American Journal of Primatology 62, 139-164.

Leigh, S.R., 2012. Brain size growth and life history in human evolution. Evolutionary Biology 39, 587-599.

Macchiarelli, R., Bondioli, L., Debenath, A., Mazurier, A., Tournepiche, J.F., Birch, W., Dean, M.C., 2006. How Neanderthal molar teeth grew. Nature 444, 748-751.

Mahoney, P., Miskiewicz, J., Pitfield, R., Schlecht, S.H., Deter, C., Guatelli-Steinberg, D., 2016. Biorhythms, deciduous enamel thickness, and primary bone growth: a test of the HaversHalberg Oscillation hypothesis. Journal of Anatomy 228, 919-928.

Martin, L.B., 1983. The relationships of the later Miocene Hominoidea. Ph.D. Dissertation, University College London.

McHenry, H., Coffing, K., 2000. Australopithecus to Homo: transformations in body and mind. Annual Review of Anthropology 29, 125-146.

Modesto-Mata, M., Dean, M.C., Lacruz, R.S., Bromage, T.G., García-Campos, C., Martínez de Pinillos, M., Martín-Francés, L., Martinón-Torres, M., Carbonell, E., Arsuaga, J.L., Bermúdez de Castro, J.M., 2020. Short and long period growth markers of enamel formation distinguish European Pleistocene hominins. Scientific Reports 10, 4665.

Orme, D., Freckleton, R., Thomas, G., Petzoldt, T., Fritz, S., Isaac, N., 2010. Caper: comparative analyses of phylogenetics and evolution in $\mathrm{R}$.

http://cran.project.org/web/packages/caper/vignettes/caper.pdf. 
Ponce de León, M.S., Zollikofer, C.P.E., 1999. New evidence from Le Moustier 1: Computerassisted reconstruction and morphometry of the skull. Anatomical Record 254, 474-489.

Pontzer, H., 2012. Ecological energetics in early Homo. Current Anthropology 53, S346-358.

Pontzer, H., 2017. The crown joules: energetics, ecology, and evolution in humans and other primates. Evolutionary Anthropology 26, 12-24.

Reiches, M.W., Ellison, P.T., Lipson, S.F., Sharrock, K.C., Gardiner, E., Duncan, L.G., 2009. Pooled energy budget and human life history. American Journal of Human Biology 21, 421-429.

R Core Team, 2018. R: A language and environment for statistical computing. R Foundation for Statistical Computing, Vienna.

Reid, D.J., Dean, M.C., 2006. Variation in modern human enamel formation times. Journal of Human Evolution 50, 329-346.

Reid, D.J., Ferrell, R.J., 2006. The relationship between number of striae of Retzius and their periodicity in imbricational enamel formation. Journal of Human Evolution 50, 195-202.

Reid, D.J., Schwartz, G.T, Dean, M.C., Chandresakera, M.S., 1998. A histological reconstruction of dental development in the common chimpanzee, Pan troglodytes. Journal of Human Evolution 35, 427-448.

Richmond, B., Jungers, W.L., 1995. Size variation and sexual dimorphism in Australopithecus afarensis and living hominoids. Journal of Human Evolution 29, 229-245.

Rosas, A., Rios, L., Estalrrich, A., Liversidge, H., Garcia-Tabernero A., Huguet, R., Cardoso, H., Bastir, M., Lalueza-Fox, C., Rasilla, M., Dean, M.C., 2017. The growth pattern of Neandertals, reconstructed from a juvenile skeleton from El Sidrón (Spain). Science 357, 1282-1287.

Ruff, C.B., Trinkaus, E., Holliday, T.W., 1997. Body mass and encephalization in Pleistocene Homo. Nature 387, 173-176.

Schultz, A.H., 1960. Age changes in primates and their modification in man. In: Tanner, J.M. (Ed.), Human Growth. Pergamon, New York, pp. 1-20.

Schwartz, G.T., 2012. Growth, development, and life history throughout the evolution of Homo. Current Anthropology 53, S395-S408.

Schwartz, G.T., Reid, D.J., Dean, M.C., 2001. Developmental aspects of sexual dimorphism in hominoid canines. International Journal of Primatology 22, 837-860.

Schwartz, G.T., Miller, E.R., Gunnell, G.F., 2005. Developmental processes and canine dimorphism in primate evolution. Journal of Human Evolution 48, 97-103.

Schwartz, G.T., Reid, D.J., Dean, M.C., Zihlman, A.L., 2006. A faithful record of stressful life events recorded in the dental development record of a juvenile gorilla. International Journal of Primatology 27, 1201-1219.

Smith, B.H., \& Tompkins, R.L., 1995. Toward a life history of the Hominidae. Annual Review of Anthropology 24, 257-279.

Smith, T.M., 2004. Incremental development of primate dental enamel. Ph.D. dissertation, State University of New York Stony Brook.

Smith, T.M., 2008. Incremental dental development: methods and applications in hominoid evolutionary studies. Journal of Human Evolution 54, 205-224.

Smith, T.M., 2016. Dental development in living and fossil orangutans. Journal of Human Evolution 94, 92-105.

Smith, T.M., Reid, D.J., Dean, M.C., Olejiczak, A.J., Ferrell, R.J., Martin, L.B., 2007a. New perspectives on chimpanzee and human molar crown development. In: Bailey, S.E., 
Hublin, J.-J. (Eds.), Dental Perspectives on Human Evolution. Springer, New York, pp. 177-192.

Smith, T.M., Tafforeau, P., Reid, D.J., Grun, R., Eggins, S., Boutakiout, M., Hublin, J.J. 2007 b. Earliest evidence of modern human life history in North African early Homo sapiens. Proceedings of the National Academy of Sciences USA 104, 6128-6133.

Smith, T.M., Toussaint, M., Reid, D.J., Olejniczak, A.J., Hublin, J.J. 2007c. Rapid dental development in a Middle Paleolithic Belgian Neanderthal. Proceedings of the National Academy of Sciences USA 104, 20220-20225.

Smith, T.M., Harvati, K., Olejniczak, A.J., Reid, D.J., Hublin, J.J., Panagopoulou, E., 2009 a. Brief communication: Dental development and enamel thickness in the Lakonis Neanderthal molar. American Journal of Physical Anthropology 138, 112-118.

Smith, T.M., Olejniczak, A.J., Kupczik, K., Lazzari, V., Vos, J., Kullmer, O., Schrenk, F., Hublin, J.-J., Jacob, T., Tafforeau, P., 2009b. Taxonomic assessment of the Trinil molars using non-destructive 3D structural and developmental analysis. PaleoAnthropology. 2009, 117-129.

Smith, T.M., Tafforeau, P., Reid, D.J., Pouech, J., Lazzari, V., Zermeno, J.P., Guatelli-Steinberg, D., Olejniczak, A.J., Hoffman, A., Radovcic, J., Masrour, M., Toussaint, M., Stringer, C., Hublin, J.J., 2010. Dental evidence for ontogenetic differences between modern humans and Neanderthals. Proceedings of the National Academy of Sciences USA 107, 2092320928.

Smith, T.M., Tafforeau, P., Le Cabec, A., Bonnin, A., Houssaye, A., Pouech, J., Moggi-Cecchi, J., Manthi, F., Ward, C.V., Makaremi, M., Menter, C.G., 2015. Dental ontogeny in Pliocene and early Pleistocene hominins. PLoS One 10, e0118118.

Smith, T.M., Houssaye, A., Kullmer, O., Le Cabec, A., Olejniczak, A.J., Schrenk, F., de Vos, J., Tafforeau, P., 2018. Disentangling isolated dental remains of Asian Pleistocene hominins and pongines. PLoS One 13, e0204737.

Spoor, F., Wood, B., Zonneveld, F., 1994. Implications of early hominid labyrinthine morphology for evolution of human bipedal locomotion. Nature 369, 645-648.

Spoor, F., Hublin, J.J., Braun, M., Zonneveld, F., 2003. The bony labyrinth of Neanderthals. Journal of Human Evolution 44, 141-165.

Spoor F., Garland, T., Krovitz, G., Ryan, T.M., Silcox, M., Walker, A., 2007. The primate semicircular canal system and locomotion. Proceedings of the National Academy of Sciences USA 104, 10808-10812.

Symonds, M.R.E., Blomberg, S.P., 2014. A primer on phylogenetic generalized least squares. In: Garamszegi, L.Z. (Ed.), Modern Phylogenetic Comparative Methods and Their Application in Evolutionary Biology. Springer, Berlin, pp. 105-130.

Trinkaus, E., Churchill, S.E., Ruff, C.B., Vandermeersch, B., 1999. Long bone shaft robusticity and body proportions of the Saint-Cesaire 1 Chatelperronian Neanderthal. Journal of Archaeological Science 26, 753-773.

Walker, A., Ryan, T.M., Silcox, M.T., Simons, E.L., Spoor, F., 2008. The semicircular canal system and locomotion: the case of extinct lemuroids and lorisoids. Evolutionary Anthropology 17, 135-145.

Ward, C.V., Leakey, M.G., Walker, A., 2001. Morphology of Australopithecus anamensis from Kanapoi and Allia Bay, Kenya. Journal of Human Evolution 41, 255-368.

Xing, S., Tafforeau, P., O’Hara, M., Modesto-Mata, M., Martin-Frances, L., Martinon-Torres, M., Zhang, L., Schepartz, L.A., Bermudez de Castro, J.M., Guatelli-Steinberg, D., 2019. 
First systematic assessment of dental growth and development in an archaic hominin (genus, Homo) from East Asia. Science Advances 5, eaau0930.

\section{Figure captions}

Figure 1. Enamel increments in an orangutan (Pongo pygmaeus pygmaeus) molar, adapted from Hogg et al. (2015). Dentine is toward the right, enamel surface to the left. Cusp tips are toward the top. In the inset, individual enamel prisms (yellow arrow) run from the dentine to the enamel surface, with daily cross striations (yellow hash-marks) running across prism long axes. The yellow hash-marks merely serve as visual indicators to help the eye identify the position and orientation of daily cross-striations. Striae of Retzius (white arrows) run obliquely from outer enamel to the enamel-dentine junction. RP (11 days, as published originally in Kelley and Schwartz, 2010) can be determined by either visually counting cross striations between successive striae of Retzius, and/or by dividing DSR by average stria of Retzius breadth within a particular tooth region. Ideally, both techniques should be cross-referenced against each other when generating RP data.

Figure 2. Boxplot depicting Retzius periodicity (RP) variation for each species in our sample. The thick black lines represent the median, the boxes represent the first and third quartiles, the whiskers represent the $5^{\text {th }}$ and $95^{\text {th }}$ percentiles.

Figure 3. Results for PGLS regression of $\ln$ Retzius periodicity (RP) against ln body mass. Slope equation: $\mathrm{y}=0.10 \mathrm{x}+1.56, \mathrm{R}^{2}=0.38$.

Figure 4. Results for PGLS regression of ln Retzius periodicity (RP) against ln mean semicircular canal radius (SCR). Slope equation: $\mathrm{y}=-1.64 \mathrm{x}+2.89, \mathrm{R}^{2}=0.45$. 